\title{
Graphical properties of the bipartite graph of $\operatorname{Spec}(\mathbb{Z}[x]) \backslash\{0\}$
}

\author{
Christina Eubanks-Turner ${ }^{1 *}$, Aihua $\mathrm{Li}^{2 * *}$ \\ 1. Department of Mathematics, Loyola Marymount University, Los Angeles, California \\ 2. Department of Mathematical Sciences, Montclair State University, Montclair, New Jersey
}

\begin{abstract}
Consider $\operatorname{Spec}(\mathbb{Z}[x])$, the set of prime ideals of $\mathbb{Z}[x]$ as a partially ordered set under inclusion. By removing the zero ideal, we denote $G_{\mathbb{Z}}=\operatorname{Spec}(\mathbb{Z}[x]) \backslash\{0\}$ and view it as an infinite bipartite graph with the prime ideals as the vertices and the inclusion relations as the edges. In this paper, we investigate fundamental graph theoretic properties of $G_{\mathbb{Z}}$. In particular, we describe the diameter, circumference, girth, radius, eccentricity, vertex and edge connectivity, and cliques of $G_{\mathbb{Z}}$. The complement of $G_{\mathbb{Z}}$ is investigated as well.
\end{abstract}

2010 MSC: 05C25, 05C63, 06A11

Keywords: Bipartite graph, Prime spectrum, Poset, Ring theory

\section{Introduction}

Throughout, $R$ is a commutative Noetherian ring with identity 1 . The prime spectrum of $R, \operatorname{Spec}(R)$, is the partially ordered set (poset) of prime ideals of $R$ ordered by inclusion. Considerable research has been done in order to determine which partially ordered sets (posets) arise as spectra of two-dimensional integral domains, such as, spectra of polynomial and power series rings [5-8, 10, 11].

In the past, diagrams were used to represent the prime spectra of certain rings. We refer to these diagrams as spec graphs. For example, in 1989, William Heinzer and Sylvia Wiegand used spec graphs to help illustrate the relationships of the elements of $\operatorname{Spec}(R[y])$, where $R$ is a semi-local countable onedimensional Noetherian domain and $y$ is an indetereminate. Similarly, spec graphs have been used to describe certain polynomial rings and power series rings (cf. [2-4, 7, 8]). The primary aim of those papers is to find properties that the spectra satisfy as partially ordered sets, in particular those properties that determine the posets.

* E-mail: ceturner@lmu.edu, supported by the Association for Women in Mathematics.

**E-mail: lia@mail.montclair.edu 
Recently, researchers have begun to give more attention to graphs derived from the ring structure. They have also studied connections between the graph properties and the original rings. For example, in 1999 Anderson and Livingston introduced zero-divisor graphs [1]. In this direction we expand the idea of applying graph theory to spec graphs.

For a ring $R$, we consider $\operatorname{Spec}(R)$ as an undirected graph whose vertices are the prime ideals of $R$ and two distinct vertices $p$ and $q$ are adjacent if and only if either $p \subset q$ or $q \subset p$. Here the inclusion " $\subset$ " is strict, since $\operatorname{Spec}(R)$ is a poset. This implies there are no loops in the graph. We investigate spec graphs of commutative rings in a graph theoretical way, which may lead to information about the ring itself. In this paper we focus on the bipartite graph derived from $\operatorname{Spec}(\mathbb{Z}[x]) \backslash\{0\}$, which we call $G_{\mathbb{Z}}$. We give background on the poset $\operatorname{Spec}(\mathbb{Z}[x])$ in Section 2. In Section 3, we give results describing fundamental graph theoretic properties of $G_{\mathbb{Z}}$. Particularly, we show that the radius of $G_{\mathbb{Z}}$ is 3 , the diameter of $G_{\mathbb{Z}}$ is $4, G_{\mathbb{Z}}$ has infinite circumference and infinite vertex and edge connectivity. In Section 4 we look at certain interesting subgraphs of $G_{\mathbb{Z}}$ and describe properties related to those subgraphs, in particular, completeness. We also show that the complement, $\overline{G_{\mathbb{Z}}}$, retains certain properties similar to those of $G_{\mathbb{Z}}$, although $\overline{G_{\mathbb{Z}}}$ is not bipartite.

\section{Overview of $\operatorname{Spec}(\mathbb{Z}[x])$}

Definition 2.1. Let $U$ be a partially ordered set with a unique minimal element $u_{0}$. The height of an element $u$ in $U, \operatorname{ht}(u)$, is the length of the longest chain to $u$ from $u_{0}$. We denote the dimension of the set $U$ by $\operatorname{dim}(U)=\max \{\operatorname{ht}(u) \mid u \in U\}$. The set of height-i elements of $U$ is denoted $H_{i}(U)=\{u \in$ $U \mid \operatorname{ht}(u)=i\}$.

Notation 2.2. For $U$ a partially ordered set and $u \in U$, we let $u^{\uparrow}=\{v \in U \mid u<v\}$ and $u^{\downarrow}=\{v \in$ $U \mid u>v\}$.

The spectrum of the polynomial ring $\mathbb{Z}[x]$ has been well described by Roger Wiegand in 1986 [11]. In [11] Roger Wiegand characterized $\operatorname{Spec}(\mathbb{Z}[x])$ as a partially ordered set satisfying five specific axioms, called CZP Axioms.

Definition 2.3. [The CZP Axioms] For any poset $U$ the following axioms are called the $\mathbf{C Z P}$ Axioms:

(P1) $U$ is countable with a unique minimal element.

(P2) U has dimension two.

(P3) Every height-one element of $U$ is below countably infinitely many height-two elements.

(P4) For each pair of distinct elements of height one, there are only finitely many height-two elements above both of them.

(P5) Given finite subsets $S$ and $T$, with $\emptyset \neq S \subset H_{1}(U)$ and $T \subset H_{2}(U)$, there is a height-one element $w$ in $U$ such that $w<m, \forall m \in T$, and whenever $m^{\prime} \in U$ is greater than both $w$ and $s$ for some $s$ in $S$, then $m^{\prime} \in T$.

As in [8], we call the element $w$ from (P5) the radical element of the pair $(S, T)$ and it can be generalized to higher dimension posets.

Definition 2.4. Let $U$ be a partially ordered set of dimension $n$. Let $\emptyset \neq S \subseteq H_{i}(U)$ and $T \subseteq H_{i+1}(U)$ with $0<i<n$. An element $w \in U$ is called a radical element if $w<m$ and $\forall m \in T$, then $w^{\uparrow} \cap s^{\uparrow} \subseteq T$, for all $s \in S$.

Thus the fifth Axiom (P5) can be restated:

(P5') Given a pair of finite subsets $S, T$ of $U$ such that $\emptyset \neq S \subset H_{1}(U)$ and $T \subset H_{2}(U)$, there exists a height-one radical element for the pair $(S, T)$. We call such a pair $(S, T)$ in $\left(\mathrm{P}^{\prime}\right)$ a $(1,2)$-pair. 


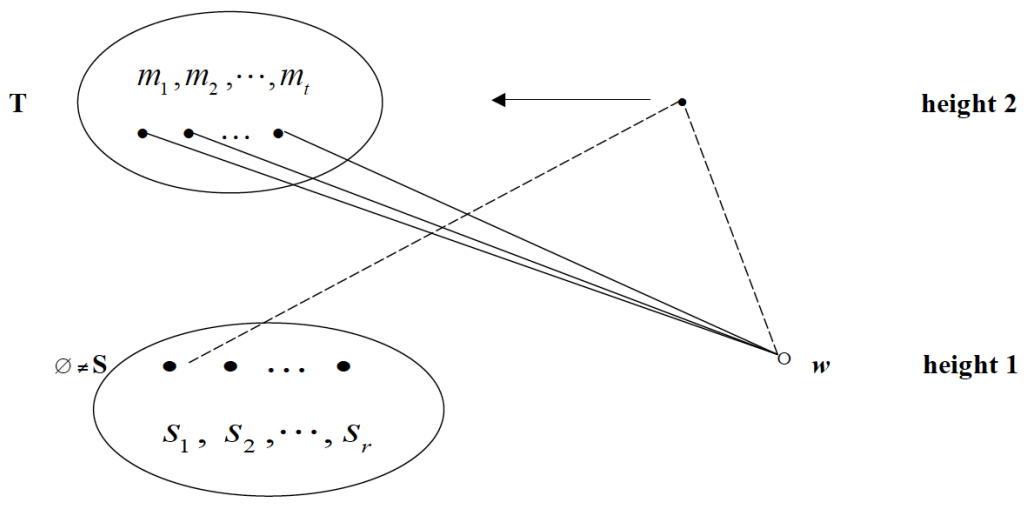

Figure 1. Illustration of axiom (P5)

Theorem 2.5. If $U$ is a poset satisfying (P1)-(P5) of Definition 2.3, then $U \cong \operatorname{Spec}(\mathbb{Z}[x])[11]$.

The following Properties from [8] are useful for our investigation.

Theorem 2.6. Let $U$ be a poset that satisfies the CZP axioms in Definition 2.3. Then

1. If $w$ is a radical element for a $(1,2)$-pair $(S, T)$, then $w \notin S$.

2. If $S \subseteq S^{\prime} \subseteq H_{1}(U)$ and $T \subseteq T^{\prime} \subseteq H_{2}(U)$, where $t \ngtr s, \forall t \in T^{\prime}-T, \forall s \in S \neq \emptyset$, then a radical element $w$ for $\left(S^{\prime}, T^{\prime}\right)$ is also a radical element for $(S, T)$.

3. In $\operatorname{Spec}(\mathbb{Z}[x])$, any $(1,2)$-pair $(S, T)$ has infinitely many radical elements.

Next we give a proposition useful for our investigation, which is related to Theorem 2.6.

Proposition 2.7. Let $U$ be a poset that satisfies the CZP axioms.

1. $\forall u \in H_{1}(U)$, there exists infinitely many $u^{\prime} \in H_{1}(U)$ such that $u^{\uparrow} \cap u^{\prime \uparrow}=\emptyset$.

2. Every height-two element of $U$ has infinitely many height-one elements below it.

3. Every height-two element of $U$ has infinitely many height-one elements not below it.

Proof. 1. follows from Theorem 2.6: any radical element $u^{\prime}$ for the $(1,2)$-pair $(\{u\}, \emptyset)$ will work. For 2. and 3 ., we use the fact that $U \cong \operatorname{Spec}(\mathbb{Z}[x])$ as posets. Consider $v=\langle f, p\rangle \in \operatorname{Spec}(\mathbb{Z}[x])$, where $p$ is a prime number and $f$ is an irreducible polynomial in $\mathbb{Z}[x]$. Then by Theorem $2.6(3)$, there are infinitely many radical elements for the $(1,2)$-pair $(\langle p\rangle, v)$ and so we have Statement 2. For all prime integers $q \neq p$, $\langle q\rangle \in H_{1}(U)$ and $\langle q\rangle \nless v$. Since there are infinitely many such $q, 3$ holds.

Theorem 2.8. The following Noetherian integral domains have spectra isomorphic to $\operatorname{Spec}(\mathbb{Z}[x])$ :

1. The polynomial ring $D[x]$, where $D$ is an order in an algebraic number field [10];

2. Two-dimensional integral domains which are finitely generated algebras over an algebraic extension of a finite field [11];

3. Finitely generated birational extensions of the polynomial ring $\mathbb{Z}[x]$ [8].

After characterizing $\operatorname{Spec}(\mathbb{Z}[x])$, Roger Wiegand gave the following conjecture: for every twodimensional finitely generated $\mathbb{Z}$-algebra $D, \operatorname{Spec}(D) \cong \operatorname{Spec}(\mathbb{Z}[x])$, c.f. [10]. This conjecture remains open. 


\section{Properties of the graph $G_{\mathbb{Z}}$}

In this section, we treat $G_{\mathbb{Z}}:=\operatorname{Spec}(\mathbb{Z}[x]) \backslash\{0\}$ as an undirected bipartite graph and investigate basic graph theory properties related to $G_{\mathbb{Z}}$. First, we define the graph as follows:

Definition 3.1. Let $U=\operatorname{Spec}(\mathbb{Z}[x])$ be the poset of the prime ideals of $\mathbb{Z}[x]$ ordered by inclusion. The graph $G_{\mathbb{Z}}=\left(V\left(G_{\mathbb{Z}}\right), E\left(G_{\mathbb{Z}}\right)\right)$ derived from $U$, with partites $H_{1}(U), H_{2}(U)$, is given by

$$
\begin{gathered}
V\left(G_{\mathbb{Z}}\right)=U \backslash\{0\}=H_{1}(U) \cup H_{2}(U) \text { and } \\
E\left(G_{\mathbb{Z}}\right)=\left\{s t \mid s \in H_{1}(U), t \in H_{2}(U), \text { and } s<t \text { in } U\right\} .
\end{gathered}
$$

All edges of $G_{\mathbb{Z}}$ have the form: st, where $s \in H_{1}(U), t \in H_{2}(U)$.

Remark 3.2. Note that since $U$ is a partially ordered set this ensures the graph $G_{\mathbb{Z}}$ is bipartite. That is, every edge of $G_{\mathbb{Z}}$ has one vertex in $H_{1}(U)$ and one vertex in $H_{2}(U)$.

We now denote $H_{i}(U)$ as $H_{i}\left(G_{\mathbb{Z}}\right)$ for $i=1,2$. Many basic graph theory properties of $G_{\mathbb{Z}}$ can be obtained from the ring theory properties of $\operatorname{Spec}(\mathbb{Z}[x])$, for example, Proposition $2.7(2)$ and (3) shown above. We investigate the connectivity, existence of cycles of different sizes, cut-edges, cut-vertices, values of girth, diameter, circumference and so on. Existence of radical elements plays an important role in the process. Here, we define the latter mentioned terms.

Definition 3.3. Let $G$ be a graph.

1. The girth of $G$ is the length of the shortest cycle contained in $G$.

2. The circumference of a graph $G$ is the longest cycle contained in $G$.

3. A cut-edge in a graph $G$ is an edge that increases the disconnectivity when removing it.

4. A cut-vertex in a graph $G$ is a vertex that increases the disconnectivity when removing it.

Theorem 3.4. $G_{\mathbb{Z}}$ has no cut-edges. Furthermore, every edge of $G_{\mathbb{Z}}$ is contained in a cycle of length 4. Furthermore, $\operatorname{girth}\left(G_{\mathbb{Z}}\right)=4$ and circumference $\left(G_{\mathbb{Z}}\right)=\infty$.

Proof. It is known that an edge is not a cut-edge if and only if it is included in a cycle. Assume $e=u v \in E\left(G_{\mathbb{Z}}\right)$, where $u \in H_{1}\left(G_{\mathbb{Z}}\right), v \in H_{2}\left(G_{\mathbb{Z}}\right)$. By Theorem 2.5 (P3), there are infinitely many elements in $H_{2}\left(G_{\mathbb{Z}}\right)$ adjacent to $u$. Choose $v^{\prime} \in H_{2}\left(G_{\mathbb{Z}}\right)$, where $v \neq v^{\prime}$, such that $u v^{\prime} \in E\left(G_{\mathbb{Z}}\right)$. By Theorem $2.7(3)$, there are infinitely many radical elements $w \in H_{1}\left(G_{\mathbb{Z}}\right)$ of the $(1,2)$-pair $\left(\{u\},\left\{v, v^{\prime}\right\}\right)$ which are adjacent to each of $v$ and $v^{\prime}$, that is, $w v, w v^{\prime} \in E\left(G_{\mathbb{Z}}\right)$. Thus we obtain infinitely many cycles of length four of the form $u v w v^{\prime} u$ including the edge $e=u v$. So $e$ is not a cut-edge. Furthermore, a cycle of length 4 must be the shortest cycle since $G_{\mathbb{Z}}$ is bipartite and there is no cycle of length 3 . Thus, $\operatorname{girth}\left(G_{\mathbb{Z}}\right)=4$.

To see that the circumference $\left(G_{\mathbb{Z}}\right)=\infty$, consider a vertex $u_{1} \in H_{2}\left(G_{\mathbb{Z}}\right)$. By Proposition 2.7 (2) $\left|u_{1}^{\downarrow}\right|=\infty$ and so there exist $u_{2} \in H_{1}\left(G_{\mathbb{Z}}\right)$ such that $u_{1} u_{2} \in E\left(G_{\mathbb{Z}}\right)$. We can choose $u_{3} \in u_{2}^{\uparrow} \backslash\left\{u_{1}\right\}$, since $\left|u_{2}^{\uparrow}\right|=\infty$ by Theorem 2.5 (P3). Note that in this setting $u_{2}, u_{4}, \ldots, u_{2 i}, \ldots$ are height-one and $u_{1}, u_{3}, \ldots, u_{2 i+1}, \ldots$ are height-two. Continuing in this manner for any $r \in \mathbb{N}$, choose a radical element $w:=u_{2 r}$ for $\left(\left\{u_{2}, \ldots, u_{2 r-2}\right\},\left\{u_{1}, \ldots, u_{2 r-1}\right\}\right)$. Note $u_{1}, \ldots, u_{2 r-1}$ are all distinct. Thus we have a cycle $u_{1} u_{2} u_{3} \cdots u_{2 r-1} u_{2 r} u_{1}$ of length $n=2 r$. Since this holds for any $n$, where $n$ is even, $\operatorname{circumference}\left(G_{\mathbb{Z}}\right)=$ $\infty$.

The next theorem states that $G_{\mathbb{Z}}$ has no finite vertex cut-set.

Theorem 3.5. Consider the graph $G_{\mathbb{Z}}$. Then 
1. Every pair of vertices are connected by infinitely many paths of length $\leq 4$.

2. $G_{\mathbb{Z}}$ is connected.

3. There is no finite vertex cut-set of $G_{\mathbb{Z}}$, that is, the vertex-connectivity of $G_{\mathbb{Z}}$ is infinite.

4. The edge-connectivity of $G_{\mathbb{Z}}$ is infinite.

Proof. For 1, if $u v \in E\left(G_{\mathbb{Z}}\right), u, v$ are connected by an edge, a path of length one. Moreover, by the proof of Theorem 3.4, uv is contained in infinitely many cycles of length 4 . Thus $u$ and $v$ are connected by infinitely many paths of length 3 . Next we assume $u, v$ are not adjacent. We consider the following cases.

Case 1: $u, v \in H_{1}\left(G_{\mathbb{Z}}\right)$.

By Theorem $2.5(\mathrm{P} 3)$ we can choose $t, t^{\prime} \in H_{2}\left(G_{\mathbb{Z}}\right)$ such that $u t, v t^{\prime} \in E\left(G_{\mathbb{Z}}\right)$ and $t \neq t^{\prime}$. Thus we take a radical element $w$ for the pair $\left(\{u, v\},\left\{t, t^{\prime}\right\}\right)$. Obviously $w \notin\{u, v\}$. So we have a path of length 4 connecting $u$ and $v$ : utwt' $v$. Note, there are infinitely many choices for such $t$ and $t^{\prime}$.

Case 2: $u, v \in H_{2}\left(G_{\mathbb{Z}}\right)$.

By Proposition $2.7(2)$, there exist $s \in H_{1}\left(G_{\mathbb{Z}}\right)$ with $s u \in E\left(G_{\mathbb{Z}}\right)$. Similarly, there are infinitely many radical elements $w$ for the pair $(\{s\},\{u, v\})$ and we have the path $u w v$, of length 2 .

Case 3: $u \in H_{1}\left(G_{\mathbb{Z}}\right)$ and $v \in H_{2}\left(G_{\mathbb{Z}}\right)$.

By Theorem 2.5 (P3), $\exists t \in u^{\uparrow} \backslash\{v\}$. There are infinitely many radical elements $w$ for the pair $(\{u\},\{t, v\})$, which builds infinitely many paths of the form $u t w v$ of length 3 .

Thus, 1 holds. The above also implies that every two vertices of $G_{\mathbb{Z}}$ is connected. Thus $G_{\mathbb{Z}}$ is connected and statement 2 is true.

To see 3 , let $U^{\prime}$ be any finite subset of $V\left(G_{\mathbb{Z}}\right)$ and consider $u, v \in V\left(G_{\mathbb{Z}}\right) \backslash U^{\prime}$. By using the above proof for 1 , we can obtain infinitely many paths from $u$ to $v$, not using any vertex from $U^{\prime}$. Thus $u$ and $v$ are connected in $G_{\mathbb{Z}} \backslash U^{\prime}$ and so $U^{\prime}$ is not a vertex-cut set of $G_{\mathbb{Z}}$.

Item 3 implies 4 by the well-known fact that for any graph $G$, vertex connectivity of $G \leq$ edge connectivity of $G$, see [9].

Next we give some basic concepts of graph theory and explore these parameters for $G_{\mathbb{Z}}$.

Definition 3.6. Let $G$ be a graph.

1. The distance between two vertices $u, v$ of $G$ is the number of edges in a shortest path connecting them. We denote the distance of $u$ and $v$ as $d(u, v)$.

2. The eccentricity of a vertex $v$, ecc $(v)$, is the greatest distance from $v$ to any other vertex.

3. The radius of a graph is the minimum eccentricity over all vertices in the graph. We denote the radius of $G$ as $\operatorname{rad}(G)$. A central vertex in a graph is a vertex that achieves the radius.

4. The diameter of a graph is the maximum eccentricity over all the vertices in the graph. That is, it is the greatest distance over all pairs of vertices. We denote the diameter of a graph $G$ as diam $(G)$. A peripheral vertex in a graph is a vertex that achieves the diameter.

Theorem 3.7. $G_{\mathbb{Z}}$ satisfies the following properties:

1. For $u, v \in V\left(G_{\mathbb{Z}}\right), d(u, v) \leq 4$.

2. $\operatorname{ecc}(v)= \begin{cases}3, & \text { if } v \in H_{2}\left(G_{\mathbb{Z}}\right) \\ 4, & \text { if } v \in H_{1}\left(G_{\mathbb{Z}}\right)\end{cases}$

3. $\operatorname{rad}\left(G_{\mathbb{Z}}\right)=3$. 
4. $\operatorname{diam}\left(G_{\mathbb{Z}}\right)=4$.

5. $H_{1}\left(G_{\mathbb{Z}}\right)=\left\{\right.$ all peripheral vertices of $\left.G_{\mathbb{Z}}\right\}$ and $H_{2}\left(G_{\mathbb{Z}}\right)=\left\{\right.$ all central vertices of $\left.G_{\mathbb{Z}}\right\}$.

6. $G_{\mathbb{Z}}$ has no finite maximal path.

Proof. 1. and 2. follow from the proof of Theorem 3.5. It is straightforward that 2. implies 3,4 and 5. To see 6 ., let $P_{n}=u_{1} \ldots u_{n+1}$ be a path of length $n \geq 1$. If $u_{n+1} \in H_{1}\left(G_{\mathbb{Z}}\right)$, we choose $u_{n+2} \in u_{n+1}^{\uparrow} \backslash\left\{u_{1}, \ldots, u_{n}\right\}$ to extend $P_{n}$ into a path $u_{1} \ldots u_{n+1} u_{n+2}$ of length $n+1$. The existence of $u_{n+2}$ is based on the fact that $\left|u_{n+1}^{\uparrow}\right|=\infty$ by Theorem 2.5 (P3). If $u_{n+1} \in H_{2}\left(G_{\mathbb{Z}}\right), \exists u_{n+2} \in u_{n+1}^{\downarrow} \backslash\left\{u_{1}, \ldots, u_{n}\right\}$ by Proposition $2.7(2)$. A longer path is produced again: $u_{1} \ldots u_{n+1} u_{n+2}$.

Another topic that generates a lot of interest in graph theory is the concept of graph matchings. Below we define what a matching is and show that $G_{\mathbb{Z}}$ has a perfect matching.

Definition 3.8. A matching in a graph $G$ is a set of pair-wise vertex disjoint edges. A perfect matching is a matching which covers all vertices of the graph. That is, every vertex of the graph is incident to exactly one edge of the matching.

Theorem 3.9. $G_{\mathbb{Z}}$ has a perfect matching.

Proof. We build a matching step by step that can cover all the vertices of $G_{\mathbb{Z}}$. Start with any edge $u_{1} v_{1}$ of $G_{\mathbb{Z}}$, which is a matching of size 1 , where $u_{1} \in H_{1}\left(G_{\mathbb{Z}}\right)$ and $v_{1} \in H_{2}\left(G_{\mathbb{Z}}\right)$. Call it $M_{1}=\left\{u_{1} v_{1}\right\}$. We then list all the rest of the vertices of $H_{1}\left(G_{\mathbb{Z}}\right)$ in a countable way: $u_{2}, u_{3}, \ldots$. Similarly, we build the countable list for the rest of the vertices of $H_{2}\left(G_{\mathbb{Z}}\right): v_{2}, v_{3}, \ldots$. Next we pick $u_{2} \in H_{1}\left(G_{\mathbb{Z}}\right)$ and choose $v_{2}^{\prime}$ from $u_{2}^{\uparrow} \backslash\left\{v_{1}\right\}$. This is doable because $\left|u_{2}^{\uparrow}\right|=\infty$. Now we have a matching $M_{2}=\left\{u_{1} v_{1}, u_{2} v_{2}^{\prime}\right\}$ of size 2. In the next step, we will pick $v_{2}$ and find $u_{3}^{\prime} \in v_{2}^{\downarrow} \backslash\left\{u_{1}, u_{2}\right\}$ to extend the mating $M_{2}$ into $M_{3}=\left\{u_{1} v_{1}, u_{2} v_{2}^{\prime}, v_{2} u_{3}^{\prime}, \ldots\right\}$. Continue picking vertices alternatively from the countable lists of the two partites to enlarge the matching that eventually can cover all the vertices of $G_{\mathbb{Z}}$.

\section{Completeness in $G_{\mathbb{Z}}$ and the complement of $G_{\mathbb{Z}}$}

A complete bipartite graph is a bipartite graph where every vertex of the first partite is adjacent to every vertex of the second set. We adopt the commonly used notation $K_{m, n}$ for the complete bipartite graph with vertex partitions of size $m$ and $n$. Note that for any $n \in \mathbb{N}, K_{1, n} \subseteq G_{\mathbb{Z}}$, by (P3) Theorem 2.5. Also we consider the complement graph of $G_{\mathbb{Z}}$ and give some interesting properties of it.

\subsection{Completeness}

Here, we give a theorem showing that there are infinitely many finite complete subgraphs of $G_{\mathbb{Z}}$.

Theorem 4.1. For every $m, n \in \mathbb{N}$, there are countably infinitely many subgraphs of $G_{\mathbb{Z}}$ which are isomorphic to $K_{m, n}$.

Proof. Without loss of generality, assume $m \leq n$. We first consider the corresponding situation in $\operatorname{Spec}(\mathbb{Z}[x])$. Let $p_{1}, p_{2}, \ldots, p_{n}$ be distinct prime numbers. Then $x^{i}+p_{1} p_{2} \ldots p_{n}$ is irreducible in $\mathbb{Z}[x], \forall i, 1 \leq i \leq n$. So $\left\langle x^{i}+p_{1} p_{2} \ldots p_{n}\right\rangle \in H_{1}(\operatorname{Spec}(\mathbb{Z}[x]))$, for each $i$. Let $S=\left\{\left\langle x^{i}+p_{1} p_{2} \ldots p_{n}\right\rangle\right\}_{i=1}^{n}$ and $T=\left\{\left\langle x, p_{j}\right\rangle\right\}_{j=1}^{m} \subseteq H_{2}(\operatorname{Spec}(\mathbb{Z}[x]))$. Then $\left\langle x^{i}+p_{1} p_{2} \ldots p_{n}\right\rangle \subset\left\langle x, p_{j}\right\rangle$, for all $i, j, 1 \leq i \leq n, 1 \leq j \leq m$. Thus the corresponding subgraph of $G_{\mathbb{Z}}$ induced by $S \cup T$ is complete and isomorphic to $K_{m, n}$. Since there are infinitely many choices for such $p_{1}, p_{2}, \ldots, p_{n}$, we have infinitely many of these subgraphs. 
An immediate Corollary of Theorem 4.1 is that $G_{\mathbb{Z}}$ is not planar. First, recall that a graph $G$ is planar if $G$ can be embedded into a plane. That is, $G$ can be drawn on a plane in such a way that edges only intersect at their common vertices. Also recall that a graph is planar if and only if it does not contain $K_{3,3}$ or $K_{5}[9]$.

Corollary 4.2. $G_{\mathbb{Z}}$ is not planar and it has infinitely many non-planar subgraphs.

Proof. By Theorem 4.1, there are infinitely subgraphs of $G_{\mathbb{Z}}$ isomorphic to $K_{3,3}$. Thus we have the claim by Kuratowski's Theorem, see [9].

Next, we give a proposition that shows we can extend any finite complete bipartite subgraph of $G_{\mathbb{Z}}$ into a larger complete bipartite subgraph of $G_{\mathbb{Z}}$. Recall that for a graph $G$, a clique of a graph $G$ is a complete subgraph of $G$.

Proposition 4.3. Let $K_{n, m}$ be a complete bipartite subgraph of $G_{\mathbb{Z}}$ with partites $S \subseteq H_{1}\left(G_{\mathbb{Z}}\right)$ and $T \subseteq H_{2}\left(G_{\mathbb{Z}}\right), n, m \geq 1$.

1. Let $r_{1}$ be a positive integer. Then $K_{n, m}$ can be extended to a complete subgraph of $G_{\mathbb{Z}}$ that is isomorphic to $K_{n+r_{1}, m}$.

2. Let $S^{\uparrow}=\bigcap_{s \in S} s^{\uparrow}$. Assume $\left|S^{\uparrow}\right|=r_{0}>m$. Then for every integer $r_{2}, 0<r_{2} \leq r_{0}-m, K_{n, m}$ can be extended to a subgraph that is isomorphic to $K_{n, m+r_{2}}$.

3. $G_{\mathbb{Z}}$ has no maximum clique. However, for a fixed finite clique in $G_{\mathbb{Z}}$, all the extended cliques have a maximum partite in $\mathrm{H}_{2}\left(G_{\mathbb{Z}}\right)$.

Proof. For 1., choose a radical element $w_{1}$ for the $(1,2)$-pair $(S, T)$. Let $T^{\downarrow}=\bigcap_{t \in T} t^{\downarrow}$. Since $w_{1} \in T^{\downarrow}$, the subgraph of $G_{\mathbb{Z}}$ induced by $S \cup\left\{w_{1}\right\} \cup T$ is a complete bipartite subgraph. Continuing in this process, we can choose distinct $w_{1}, w_{2}, \ldots, w_{r_{1}}$ not in $S \cup T$ such that $S \cup\left\{w_{1}, w_{2}, \ldots, w_{r_{1}}\right\} \cup T$ generates a clique of $G_{\mathbb{Z}}$ isomorphic to $K_{n+r_{1}, m}$. To see 2 , we can choose $T_{0} \subseteq S^{\uparrow} \backslash T$ with $\left|T_{0}\right|=r_{2}$. Then $S \cup T_{0} \cup T$ will induce a clique of $G_{\mathbb{Z}}$ which is isomorphic to $K_{n, m+r_{2}}$.

For 3, without loss of generality, we assume $S$ has at least two vertices. From the proof of part 2, any added vertex from $H_{2}\left(G_{\mathbb{Z}}\right)$ in an enlarged clique must be in $S^{\uparrow} \backslash T$, which is finite by the CZP Axiom (P4) since $|S| \geq 2$. Thus only finitely many vertices from $H_{2}\left(G_{\mathbb{Z}}\right)$ can be added to any extended clique.

\subsection{The complement of $G_{\mathbb{Z}}$}

Recall that the complement $\bar{G}$ of a graph $G$ is a graph with the same vertex set as $G$ such that two vertices $u, v$ are adjacent in $\bar{G}$ if and only if $u, v$ are not adjacent in $G$. Since $G_{\mathbb{Z}}$ is bipartite, the two partites of $G_{\mathbb{Z}}$ induce two complete subgraphs of $\overline{G_{\mathbb{Z}}}$. Proposition 4.5 asserts that $\overline{G_{\mathbb{Z}}}$ is connected. In $\overline{G_{\mathbb{Z}}}$, we denote $N_{\overline{G_{\mathbb{Z}}}}(u)=\left\{v \in V\left(G_{\mathbb{Z}}\right) \mid u v \in E\left(\overline{G_{\mathbb{Z}}}\right)\right\}=\left\{v \in V\left(G_{\mathbb{Z}}\right) \mid u v \notin E\left(G_{\mathbb{Z}}\right)\right\}$.

Proposition 4.4. For every $u \in V\left(G_{\mathbb{Z}}\right),\left|N_{\overline{G_{\mathbb{Z}}}}(u)\right|=\infty$.

Proof. Case 1. $u \in H_{1}\left(G_{\mathbb{Z}}\right)$. Note that $N_{\overline{G_{\mathbb{Z}}}}(u)=H_{2}\left(G_{\mathbb{Z}}\right) \backslash u^{\uparrow}$. Assume $N_{\overline{G_{\mathbb{Z}}}}(u)$ is finite. Then $T=H_{2}\left(G_{\mathbb{Z}}\right) \backslash u^{\uparrow}$ is finite. Let $S=\{u\}$ and consider the (1,2)-pair $(S, T)$ in $G_{\mathbb{Z}}$. Since both $S, T$ are finite and $S \neq \emptyset$, there exists a radical element $w$ for the pair $(S, T)$. Also since $w \in H_{1}\left(G_{\mathbb{Z}}\right),\left|w^{\uparrow}\right|=\infty$, and $H_{2}\left(G_{\mathbb{Z}}\right)=u^{\uparrow} \cup T, \exists m \in w^{\uparrow} \cap u^{\uparrow}$ which implies $m \notin T$, contradiction.

Case 2. $u \in H_{2}\left(G_{\mathbb{Z}}\right)$. By Proposition $1(3)$, there are infinitely many vertices in $H_{1}\left(G_{\mathbb{Z}}\right)$ not adjacent to $u$ in $G_{\mathbb{Z}}$ thus adjacent to $u$ in $\overline{G_{\mathbb{Z}}}$.

Thus in both cases, $\left|N_{\overline{G_{\mathbb{Z}}}}(u)\right|=\infty$. 
Proposition 4.5. The complement $\overline{G_{\mathbb{Z}}}$ is connected and both the vertex and edge connectivity of $\overline{G_{\mathbb{Z}}}$ are infinite.

Proof. It is obvious that $\overline{G_{\mathbb{Z}}}$ is made of two complete subgraphs induced from the vertex sets $H_{1}\left(G_{\mathbb{Z}}\right)$ and $H_{2}\left(G_{\mathbb{Z}}\right)$ and there are infinitely many edges connecting vertices between the two cliques. Thus $\overline{G_{\mathbb{Z}}}$ is connected.

To see that $\overline{G_{\mathbb{Z}}}$ has infinite vertex-connectivity, let $U^{\prime}$ be a finite vertex cut set and consider any two vertices $u, v \in V\left(G_{\mathbb{Z}}\right) \backslash U^{\prime}$. We show that $u$ and $v$ are connected in $\overline{G_{\mathbb{Z}}}$. Since each of $H_{1}\left(G_{\mathbb{Z}}\right), H_{2}\left(G_{\mathbb{Z}}\right)$ induces the empty graph in $G_{\mathbb{Z}}$, we have that each induces a complete subgraph of $\overline{G_{\mathbb{Z}}}$. It is sufficient to assume $u \in H_{1}\left(G_{\mathbb{Z}}\right)$ and $v \in H_{2}\left(G_{\mathbb{Z}}\right)$. By Proposition 4.4 since $\left|N_{\overline{G_{\mathbb{Z}}}}(v)\right|=\infty$, and $\left|U^{\prime}\right|<\infty$, we can choose $w \in N_{\overline{G_{\mathbb{Z}}}}(v) \backslash U^{\prime}$ to make a cycle uwvu in $G_{\mathbb{Z}}$. Since there are infinitely many such $w, v$ and $u$ are connected in $V\left(\overline{G_{\mathbb{Z}}}\right) \backslash U^{\prime}$, we have $U^{\prime}$ is not a cut-set of $\overline{G_{\mathbb{Z}}}$. Thus, $\overline{G_{\mathbb{Z}}}$ has infinite vertex-connectivity. The latter implies infinite edge-connectivity, by the well-known fact that for any graph $G$, vertex connectivity of $G \leq$ edge connectivity of $G$, see [9].

\section{What does the graph $G_{\mathbb{Z}}$ reveal about the ring $\mathbb{Z}[x]$ ?}

Much research has been done in describing the structures of rings whose spectra are isomorphic to $\operatorname{Spec}(\mathbb{Z}[x])$ as posets. The work in this paper gives a set of graph theoretic properties of the derived graph $G_{\mathbb{Z}}$ by $\operatorname{Spec}(\mathbb{Z}[x]) \backslash\{0\}$ as an infinite bipartite graph. Is it shown that $G_{\mathbb{Z}}$ is a graph with many good properties. Although it is infinite, it has fairly small graph measurements such as girth, diameter, radius, etc. It contains many different types of finite bipartite subgraphs such as perfect matching, cliques of any size, etc. It is natural to ask: "What does the graph $G_{\mathbb{Z}}$ reveal about the ground rings represented by $\mathbb{Z}[x]$ ?" In this regard, we give some descriptions from the ring theoretic point of view about any ring whose spectrum satisfies the CZP Axioms, based on the graph theory results obtained in earlier sections of this paper.

Proposition 5.1. Let $D$ be a ring such that $\operatorname{Spec}(D)$ satisfies the CZP axioms given in Definition 2, as a poset. Then the following are true:

1. Assume $p_{1}, p_{2}$ are two co-maximal height-1 prime ideals $\left(\right.$ so $\left.p_{1}+p_{2}=D\right)$. Then there exist a height-1 prime $p$ and two maximal ideals $m_{1}, m_{2}$ such that $p_{i}+p \subseteq m_{i}$ for $i=1,2$.

2. For any pair $(p, m)$ of prime ideals, where $p$ is of height one, $m$ is maximal, and $p \not \subset m$, there exisit a height-1 prime $p^{\prime}$ and a maximal ideal $m^{\prime}$ such that $p \subseteq m^{\prime}$ and $p^{\prime} \subseteq m \cap m^{\prime}$.

3. We can order all the height-1 ideals and all the maximal ideals as two corresponding countable lists:

$$
\begin{aligned}
& H_{2}(\operatorname{Spec}(D)): m_{1}, m_{2}, \ldots, m_{n}, \ldots \\
& H_{1}(\operatorname{Spec}(D)): p_{1}, p_{2}, \ldots, p_{n}, \ldots
\end{aligned}
$$

such that for each $i, p_{i} \subseteq m_{i}$.

4. For any positive integers $n$ and $r$, there are $n$ height-1 prime ideals $p_{1}, p_{2}, \ldots, p_{n}$ and $r$ maximal ideals $m_{1}, m_{2}, \ldots, m_{r}$ such that $p_{i} \subseteq m_{j}$ for all $i, j$ where $1 \leq i \leq n$ and $1 \leq j \leq r$.

Proof. Statement 1 is immediately from Theorem 3.5 (1) (refer to Case 1 in the proof). Statement 2 is also by Theorem 3.5 (1) (refer to Case 3 in the proof). Statement 3 is from Theorem 3.9: $G_{\mathbb{Z}}$ has a perfect matching. The last statement follows directly from the existence of cliques of any size in $G_{\mathbb{Z}}$ (Theorem 4.1). 


\section{Conclusion}

This research shows that graphs derived from ring spectra may provide rich structural properties and may contain various types of graphs of interest, thus worthy of study. The graphic properties may tell something about the ring structure itself and vice versa. In the future, we will examine more types of spectra and apply more advanced graph theory techniques, such as graph polynomials, in the study.

\section{References}

[1] D. F. Anderson and P. Livingston, The zero-divisor graph of a commutative ring, J. Algebra, 217, 434-447, 1999.

[2] E. Celikabs and C. Eubanks-Turner, The Projective Line over the Integers, Progress in Commutative Algebra II: Ring Theory, Homology, and Decompositions, 221-240, De Gruyter, 2012.

[3] C. Eubanks-Turner, M. Luckas, S. Saydam, Prime ideals in Birational extensions of two-dimensional power series rings, Communications in Algebra, 41(2), 703-735, 2013.

[4] W. Heinzer, C. Rotthaus, S. Wiegand, Mixed polynomial/power series rings and relations among their spectra, Multiplicative ideal theory in commutative algebra, Springer, New York, 227-242, 2006.

[5] W. Heinzer, S. Wiegand, Prime ideals in two-dimensional polynomial rings, Proc. Amer. Math. Soc., 577-586, 1989.

[6] W. Heinzer, S. Wiegand, Prime ideals in polynomial rings over one-dimensional domains, Trans. Amer. Math. Soc., 347(2), 639-650, 1995.

[7] A. Li, S. Wiegand, The Polynomial Behavior of Prime Ideals in Polynomial Rings and the Projective Line over $\mathbb{Z}$, Factorization in Integral Domains, Lecture Notes in Pure and Applied Mathematics, 189(3), 383-400, 1997.

[8] A. Li, S. Wiegand, Prime ideals in two-dimensional domains over the integers, J. Pure Appl. Algebra, 130(3), 313-324, 1998.

[9] D. West, Introduction to Graph Theory, Prentice Hall, Upper Saddle River, NJ, 2001.

[10] R. Wiegand, Homeomorphisms of affine surfaces over a finite field, J. London Math. Soc., (2), 18(1), 28-32, 1978.

[11] R. Wiegand, The prime spectrum of a two-dimensional affine domain, J. Pure Appl. Algebra, 40(2), 209-214, 1986 